\title{
Is Bohm's Interpretation Consistent with Quantum Mechanics?
}

\author{
Michael Nauenberg
}

Department of Physics, University of California, Santa Cruz, United States. E-mail: michael@physics.ucsc.edu

Editors: Joseph B. Keller \& Danko Georgiev

Article history: Submitted on May 25, 2014; Accepted on July 26, 2014; Published on August 23, 2014.

he supposed equivalence of the conventional interpretation of quantum mechanics with Bohm's interpretation is generally demonstrated only in the coordinate representation. It is shown, however, that in the momentum representation this equivalence is not valid.

Quanta 2014; 3: 43-46.

\section{Introduction}

Recently, there has been a renewed interest in David Bohm's interpretation of non-relativistic quantum mechanics [1-4] and many pedagogical papers on this topic have appeared [5-17], while online, arXiv.org lists over 200 submissions on this topic during the past ten years. Bohm claimed that "as long as the present general form of Schrödinger's equation is retained the physical results obtained with [this] suggested alternative interpretation are precisely the same as those obtained with the usual interpretation", and that his interpretation "leads to precisely the same results for all physical processes as does the usual interpretation" [1, p.166]. Similar assertions also have been made in references [5-17], but this equivalence is usually demonstrated only in the coordinate representa-

(c) (1) This is an open access article distributed under the terms of the Creative Commons Attribution License CC-BY-3.0, which permits unrestricted use, distribution, and reproduction in any medium, provided the original author and source are credited. tion, while the implications of Bohm's interpretation in the momentum representation are usually ignored. While there have been some criticisms in the past of Bohm's interpretation of quantum mechanics [18,19], we give here an elementary proof that the momentum distribution in this interpretation differs from that in standard quantum mechanics. We show that the definition of particle velocity in this interpretation, implies that the product of mass times velocity is not equal to momentum, which is inconsistent with both classical and quantum mechanics. The word "consistent" is used here in accordance to its definition in the World English Dictionary: "A set of statements capable of all being true at the same time or under the same interpretation".

\section{Bohmian mechanics differs from conventional quantum mechanics in the momentum representation}

In Bohm's interpretation of quantum mechanics, the velocity of a particle with mass $m$ is given by

$$
\vec{v}_{B}=\vec{\nabla} S / m
$$

where $S / \hbar$ is the phase of the wave function $\psi$ obtained by solving the time dependent Schrödinger equation. According to Bohm,

$$
\vec{v}_{B}=\frac{d \vec{q}}{d t}
$$


where $\vec{q}$ is the time dependent coordinate for the position of the particle, and Equation 1 becomes a first order differential equation that determines $\vec{q}$ as a function of time $t$, given its initial value. (In his original papers, [1, 2], Bohm introduced as fundamental, the equation of motion for the acceleration $d \vec{v} / d t$. This equation can be obtained by taking the time derivative of Equation 1, but it is misleading to regard it as fundamental, because it implies that the initial velocity of the particle can be assigned arbitrarily. But given the initial position $\vec{q}$, this velocity is determined uniquely by Equation 1. Bohm's equation of motion leads to the appearance of a non-local "quantum potential" that accounts for the origin of an acceleration even when the classical potential vanishes.). But it turns out that the product $m \vec{v}_{B}$ is not equal to the canonical momentum $\vec{p}$, because $\vec{v}_{B}$ does not correspond to the velocity $\vec{v}$, that is determined in quantum mechanics by the operator

$$
\vec{v}=-\frac{i \hbar}{m} \vec{\nabla}_{q}=\frac{\vec{p}}{m}
$$

A proof of this relation is given in section 5. Setting

$$
\psi=R \exp (l S / \hbar)
$$

where $R$ is the amplitude of $\psi$, we obtain

$$
\vec{v} \psi=\left(\vec{\nabla}_{q} S / m-\imath \hbar \vec{\nabla}_{q} R / m R\right) \psi
$$

But in Bohm's definition of the particle velocity, Equation 1, only the first term on the right hand side of this equation appears. The relevance of the second term can be illustrated by considering the mean values $\langle\vec{v}\rangle$ and $\left\langle\vec{v}^{2}\right\rangle$ in this representation for $\psi$. We have

$$
\langle\vec{v}\rangle=\int d^{3} q \psi^{\dagger} \vec{v} \psi=\int d^{3} q \vec{R}^{2} \vec{\nabla} S / m=\left\langle\vec{v}_{B}\right\rangle,
$$

and

$$
\left\langle\vec{v}^{2}\right\rangle=\int d^{3} q \psi^{\dagger}(\vec{v})^{2} \psi=\left\langle\left(\vec{v}_{B}\right)^{2}\right\rangle+(\hbar / m)^{2} \int d^{3} q(\vec{\nabla} R)^{2} .
$$

Hence, Equation 7 implies that the second moment of the velocity distribution in conventional quantum mechanics differs from that obtained in Bohm's interpretation of the particle velocity, Equation 1, by the appearance of the additional term $(\hbar / m)^{2}\left\langle(\vec{\nabla} R)^{2} / R^{2}\right\rangle$ on the right hand side of this equation. Remarkably, this discrepancy is not even mentioned in any of the recent articles on Bohm's interpretation of wave mechanics [5-15]. Similar discrepancies also appear in all the higher moments of this distribution.

\section{Bohmian "osmotic velocity", "fluid flow pathlines" and particle trajectories}

To get agreement with the mean value $\left\langle\vec{v}^{2}\right\rangle$ in quantum mechanics, Equation 7, Bohm's interpretation requires, in addition to the Bohmian particle velocity $\vec{v}_{B}$ given by Equation 1, the existence of an ad hoc random velocity

$$
\vec{v}_{o}=\frac{\hbar}{m R} \vec{\nabla} R,
$$

with vanishing mean value, Originally, such a contribution was introduced with an undetermined coefficient as a random velocity by D. Bohm and J. P. Vigier [20], who named it an "osmotic velocity", after a term introduced by Einstein to describe the chaotic Brownian motion. But now such a term has been abandoned in discussions of Bohmian mechanics.

In particular, for stationary solutions of the Schrödinger, the phase $S=0$, and Bohm's interpretation leads to the conclusion that the particle velocity vanishes in such a state. This conclusion is explained by invoking a quantum force due to a non-local quantum potential that supposedly balances the force due to the conventional potential that gives rise to the stationary solution. This non-classical force appears when the acceleration $d^{2} \vec{q} / d t^{2}$ is calculated by taking the time derivative of Equation 1 and Equation 2. But this result contradicts the fact that in quantum mechanics the velocity or momentum distribution for stationary solutions, given by the absolute square of the Fourier transform of $\psi$ in coordinate space, is not a delta function at $\vec{v}=0$, as is implied by Bohm's interpretation.

The trajectories obtained by integrating Bohm's first order differential equation for the particle coordinate $\vec{q}$, Equation 2, correspond to pathlines associated with the probability distribution $\rho=|\psi|^{2}$ which satisfies, like a normal fluid of density $\rho$, the continuity equation,

$$
\frac{\partial \rho}{\partial t}+\vec{\nabla}_{q} \cdot \vec{j}=0
$$

where $\vec{j}=\vec{v}_{B} \rho$ is the associated current. While pathlines provide a visualization of a fluid flow, these lines do not correspond to the actual motion of the particles composing the fluid that also can have a random component. Likewise, Bohmian pathlines serve to visualize the evolution of the probability distribution in quantum mechanics, but do not correspond to actual trajectories of elementary particles.

Recently, experiments have been made with water droplets surfing on the waves produced by the Faraday instability on the surface of an oscillating tank filled with 
a fluid [21]. The motion of these droplets mimics the suggestion of de Broigle and of Bohm that elementary particles are likewise "piloted" by the $\psi$ function of wave mechanics. In particular, it is claimed that when the waves propagate through two slits, or are confined in a "corral", the droplets satisfy statistics that are similar to those observed for particles in quantum mechanics. But such experiments only demonstrate the universality of wave propagation, and the associated pathlines, whether governed by the equations of fluid mechanics, quantum mechanics, or of other sources of waves in physics.

\section{Discussion}

In his original articles [1,2], Bohm proposed an extension of de Broigle's pilot wave theory of quantum mechancs which he asserted to be equivalent to Schrödinger's formulation of wave mechanics (together with Born's statistical interpretation). In Bohm's theory, particles move along classical trajectories with a velocity determined by the phase of Schrödinger's wave function $\psi$, satisfying a second order Newtonian-like equation of motion, but with an additional force due to a so-called "quantum potential", that is obtained from a solution of Schrödinger's equation. In his theory, the statistical character emerges from the unknown initial velocity and position of the particle that is given by the probability distribution $|\psi|^{2}$. Actually, Bohm's velocity satisfies a first order equation, and therefore only the initial position of the particle, but not its velocity, can be imposed arbitrarily.

We have shown that in Bohm's interpretation of quantum mechanics, the product of mass $m$ times the velocity $v$ of a particle does not correspond to the momentum. Hence, this interpretation is not only inconsistent with the standard formulation of quantum mechanics, but also with classical mechanics, where momentum is defined by the relation $p=m v$. But such inconsistencies were not mentioned in Bohm's original articles, and are now generally ignored in the vast literature on this subject.

\section{Appendix. The relation between velocity and momentum in non-relativistic quantum mechanics}

In quantum mechanics, the velocity $\vec{v}$, like the position $\vec{q}$ and the momentum $\vec{p}$, is an operator. It is defined by the relation

$$
\vec{v}=\frac{l}{\hbar}[H, \vec{q}]
$$

where $H$ is the hamiltonian operator, and $[a, b]=a b-$ $b a$ is the commutator of the operators $a$ and $b$. In nonrelativistic quantum mechanics,

$$
H=-\frac{\hbar^{2}}{2 m} \nabla_{q}^{2}+V(\vec{q})
$$

corresponding to the time dependent Schrödinger equation

$$
\hbar \hbar \frac{\partial \psi}{d t}=H \psi
$$

Hence, substituting this expression for $H$ in Equation 10 , one finds that the velocity operator is given by

$$
\vec{v}=\frac{\vec{p}}{m}
$$

where

$$
\vec{p}=-\imath \hbar \vec{\nabla}_{q}
$$

is the momentum operator.

For an alternative derivation of the connection between the velocity and momentum operators, Equation 13, that does not presuppose the Schrödinger equation, Equation 11 and Equation 12, consider the commutation relation Equation 10 for the Hamiltonian of a free particle $H_{0}=\vec{p}^{2} / 2 m$. Then, according to the definition of velocity, Equation 10.

$$
v_{i}=\frac{l}{2 \hbar m}\left(p_{j}\left[p_{j}, q_{i}\right]+\left[p_{j}, q_{i}\right] p_{j}\right),
$$

and substituting the Heisenberg-Born commutation relation

$$
\left[p_{j}, q_{i}\right]=-\imath \hbar \delta_{i, j}
$$

leads again to Equation 13

\section{References}

[1] Bohm D. A suggested interpretation of the quantum theory in terms of "hidden" variables I. Physical Review 1952; 85 (2): 166-179. http://dx.doi. org/10.1103/PhysRev.85.166

[2] Bohm D. A suggested interpretation of the quantum theory in terms of "hidden" variables. II. Physical Review 1952; 85 (2): 180-193. http://dx.doi, org/10.1103/PhysRev. 85.180

[3] Bohm D, Hiley BJ. The Undivided Universe: An Ontological Interpretation of Quantum Theory. New York: Routledge, 1993.

[4] Bacciagaluppi G, Valentini A. Quantum Theory at the Crossroads: Reconsidering the 1927 Solvay Conference. Cambridge: Cambridge University Press, 2009. http://arxiv.org/abs/ quant-ph/0609184 
[5] Matzkin A. Realism and the wavefunction. European Journal of Physics 2002; 23 (3): 285-294. http://dx.doi.org/ 10.1088/0143-0807/23/3/307 http: //arxiv.org/abs/quant-ph/0208018

[6] Boozer AD. Hidden variable theories and quantum nonlocality. European Journal of Physics 2009; 30 (2): 355-365. http://dx.doi.org/10.1088/ 0143-0807/30/2/015

[7] Passon O. How to teach quantum mechanics. European Journal of Physics 2004; 25 (6): 765-769. http://dx. doi.org/10.1088/0143-0807/25/6/008 http://arxiv.org/abs/quant-ph/0404128

[8] Tumulka R. Understanding Bohmian mechanics: A dialogue. American Journal of Physics 2004; 72 (9): 1220-1226. http://dx.doi.org/ 10.1119/1.1748054 http://arxiv.org/abs/ quant-ph/0408113

[9] Tumulka R. Feynman's path integrals and Bohm's particle paths. European Journal of Physics 2005; 26 (3): L11-L13. http://dx.doi.org/ 10.1088/0143-0807/26/3/L01 http://arxiv. org/abs/quant-ph/0501167

[10] Nikolić H. Would Bohr be born if Bohm were born before Born? American Journal of Physics 2008; 76 (2): 143-146. http://dx.doi.org/10.1119/ 1.2805241 http://arxiv.org/abs/physics/ 0702069

[11] Bernstein J. More about Bohm's quantum. American Journal of Physics 2011; 79 (6): 601-606. http://dx.doi.org/10.1119/1.3556713

The author asserted that "when unambiguous the predictions of the two theories [Bohmian mechanics and standard quantum mechanics] are identical."

[12] Sanz AS, Miret-Artés S. Setting up tunneling conditions by means of Bohmian mechanics. Journal of Physics A: Mathematical and Theoretical 2011; 44 (48): 485301. http://dx doi.org/10.1088/1751-8113/44/48/485301 http://arxiv.org/abs/1104.1298

[13] Sanz AS, Miret-Artés S. Quantum phase analysis with quantum trajectories: A step towards the creation of a Bohmian thinking. American Journal of Physics 2012; 80 (6): 525-533. http://dx.doi org/10.1119/1.3698324 http://arxiv.org/ abs/1104.1296

In a departure from the usual Bohmian interpretation of quantum mechanics, the authors suggest that "if the Bohmian equations are understood as hydrodynamic equations, the trajectories obtained from the equation of motion (see Equation 1 ) should not be regarded necessarily as the trajectories pursued by real particles, but rather as the streamlines associated with the quantum fluid". This article also contains a large number of references to the literature on Bohm's interpretation of quantum mechanics.

[14] Norsen T. The pilot-wave perspective on quantum scattering and tunneling. American Journal of Physics 2013; 81 (4): 258-266. http://dx.doi. org/10.1119/1.4792375 http://arxiv.org/ abs $/ 1210.7265$

[15] Beneduci R, Schroeck Jr FE. On the unavoidability of the interpretations of quantum mechanics. American Journal of Physics 2014; 82 (1): 8082. http://dx.doi.org/10.1119/1.4824797 http://arxiv.org/abs/1211.3883

[16] Goldstein S. Quantum theory without observerspart two. Physics Today 1998; 51 (4): 38-52. http: //dx.doi.org/10.1063/1.882241

The author concluded that Bohmian mechanics "agrees completely with orthodox quantum theory in its predictions. Precise and simple it involves an almost obvious incorporation of Schrödiger's equation into an entirely deterministic reformulation of quantum theory".

[17] Goldstein S. Bohmian mechanics. In: Stanford Encyclopedia of Philosophy, Zalta EN, Nodelman U, Allen C (editors), Stanford, California: Stanford University, 2012; http://plato.stanford, edu/entries/qm-bohm/

[18] Keller JB. Bohm's interpretation of the quantum theory in terms of "hidden" variables. Physical Review 1953; 89 (5): 1040-1041. http://dx.doi.org/ 10.1103/PhysRev. 89.1040

A related demonstration of the inconsistency of Bohm's interpretation of quantum mechanics was pointed out by Keller more than 60 years ago, but it has either been ignored or forgotten.

[19] van Kampen NG. The scandal of quantum mechanics. American Journal of Physics 2008; 76 (11): 989990. http://dx.doi.org/10.1119/1.2967702

[20] Bohm D, Vigier JP. Model of the causal interpretation of quantum theory in terms of a fluid with irregular fluctuations. Physical Review 1954; 96 (1): 208-216. http://dx.doi.org/10.1103/ PhysRev.96.208

[21] Harris DM, Moukhtar J, Fort E, Couder Y, Bush JWM. Wavelike statistics from pilot-wave dynamics in a circular corral. Physical Review E 2013; 88 (1): 011001.http://dx.doi.org/10.1103/ PhysRevE. 88.011001 